\title{
Bacterial diversity in goat milk from the Guanzhong area of China
}

\author{
Fuxin Zhang, ${ }^{* 1}$ Zhaoxia Wang, $\dagger^{1}$ Feiyan Lei, ${ }^{*}$ Bini Wang, ${ }^{*}$ Shuaiming Jiang,‡ Qiannan Peng,‡ \\ Jiachao Zhang, $\ddagger^{2}$ and Yuyu Shao*2* \\ *College of Food Engineering and Nutritional Science, Shaanxi Normal University, Xi'an 710119, Shaanxi, China \\ †College of Food Science and Technology, Hainan University, Haikou 570228, Hainan, China \\ ‡College of Food Science and Engineering, Inner Mongolia Agricultural University, Hohhot 010018, Inner Mongolia, China
}

\begin{abstract}
In this study, the V3 and V4 regions of the $16 \mathrm{~S}$ rRNA gene from metagenomic DNA were sequenced to identify differences in microbial diversity in raw milk of Saanen and Guanzhong goats from the Guanzhong area of China. The results showed that Proteobacteria was the predominant phylum, accounting for $71.31 \%$ of all phyla identified in milk from the 2 breeds, and Enterobacter was the predominant genus (24.69\%) within the microbial community. Microbial alpha diversity from Saanen goat milk was significantly higher than that of Guanzhong goat milk based on bioinformatic analysis of indices of Chao1, Shannon, Simpson, observed species, and the abundance-based coverage estimator. Functional genes and their likely metabolic pathways were predicted, which demonstrated that the functional genes present in the bacteria in goat milk were enriched in pathways for amino acid metabolism and carbohydrate metabolism, which represented 11.93 and $11.23 \%$ of functional genes, respectively. Physicochemical properties such as $\mathrm{pH}$, protein, fat, and AA levels were also determined and correlations made with microbial diversity. We detected a significant difference in the content of lactose and $6 \mathrm{AA}$, which were higher in Saanen milk than in Guanzhong milk, and positively correlated with microbial carbohydrate metabolism and AA metabolism. Lactococcus, Lactobacillus, Bifidobacterium, Enterococcus, and Streptococcus, which are lactose-utilizing genera, were more abundant in Saanen milk than in Guanzhong milk. Higher levels of lactose in Saanen goat milk may explain its greater microbial diversity. We also demonstrated that most of the AA metabolism-related bacterial genera (e.g., Massilia, Bacteroides, Lysobacter) were enriched in Saanen goat milk. In this research, both probiotic and pathogenic
\end{abstract}

\footnotetext{
Received May 28, 2017.

Accepted June 21, 2017.

${ }^{1}$ These authors contributed equally to this work.

${ }^{2}$ Corresponding authors: zhjch321123@163.com and icheesemaking@ yahoo.com
}

bacteria were identified in goat milk, which provided the microbial information necessary to direct the utilization of beneficial microbial resources and prevent the development of harmful organisms in goat milk.

Key words: metagenomics, 16S rRNA gene, lactic acid bacteria, psychrotrophic bacteria, PICRUSt (Phylogenetic Investigation of Communities by Reconstruction of Unobserved States)

\section{INTRODUCTION}

China has large numbers of dairy goats, which are mainly distributed in the Guanzhong area. The 2 predominant breeds of dairy goats in the Guanzhong area are Saanen and Guanzhong (Jia and Wei, 2016; Zhou et al., 2016). Goat milk is easily digested and absorbed, has a high nutritional value, and it contains many probiotics, including lactic acid bacteria (García et al., 2014). However, compared with that in cow milk, coagulation of goat milk through fermentation is more difficult due to the differences in protein composition (Gursel et al., 2016), and lactic acid bacteria sourced from goat milk may be more suitable for goat milk fermentation (Silva et al., 2016).

Identification of the microbial community present in milk is important to guarantee the safety of raw milk and milk products. However, some bacteria exist in a viable but nonculturable (VBNC) state, making it difficult to determine the entire microbial composition using culture-dependent methods. Cells in this dormant state are only metabolically active enough to sustain life; they are capable of surviving in adverse environments (e.g., low temperature or $\mathrm{pH}$, under nutrient starvation, elevated salinity) but cannot be cultivated by conventional methods (Ayrapetyan et al., 2015; Kibbee and Örmeci, 2017). Pathogens in a VBNC state have the potential to survive and subsequently recover in suitable environments, posing an undetectable risk to public health. It is therefore important to develop alternative methods to detect and control pathogens in the VBNC state in contaminated foods to ensure food safety (Ayrapetyan and Oliver, 2016). 
Metagenomics is an effective way to identify the microorganisms present in samples; it involves analysis of the total DNA from all microbes present in a given environment, including those in the VBNC state, and is independent of cultivation (De Filippis et al., 2017). The $16 \mathrm{~S}$ rRNA gene is the preferred gene for metagenomics analysis of microbial diversity rather than using whole metagenomic DNA (Yang et al., 2016). In prokaryotic cells, the 16S rRNA gene contains both conserved regions and variable regions, the latter of which can be sequenced to discriminate between microbes for bacterial phylogenetic and taxonomic identification. In this study, the variable V3 and V4 regions of the 16S rRNA genes were used to determine the microbial diversity in raw goat milk from Saanen and Guanzhong dairy goats. The nutritional components of the 2 types of milk were also evaluated for potential correlation with the microbial community. This research provides microbial information on goat milk, which is necessary to make the most of the beneficial probiotic species present and prevent or control harmful bacteria.

\section{MATERIALS AND METHODS}

\section{Sample Collection}

Milk samples were taken simultaneously from each of 3 Saanen dairy goats (S1 to S3) and 3 Guanzhong dairy goats (G1 to G3) from the same grazing area in the Guanzhong region of China, where the average temperature was $30^{\circ} \mathrm{C}$. All the goats were in the third month of lactation and samples were taken from the bulk milk $2 \mathrm{~h}$ after milking of each goat (the first 3 milk drops from each goat were discarded) and immediately placed into sterile tubes (one sample from the bulk milk from each goat), numbered, and placed in an ice box for transportation to the laboratory for extraction of the metagenomic DNA.

\section{Extraction of Metagenomic DNA of Bacteria from Goat Milk}

Good quality metagenomic DNA is essential for effective analysis of microbial diversity. As the number of microorganisms in milk samples is relatively small, each 200-mL sample of milk was concentrated for 10 $\min$ at $2,770 \times g\left(4^{\circ} \mathrm{C}\right)$. The precipitates were washed 3 times in a $0.8 \% \mathrm{NaCl}$ solution. Metagenomic DNA was extracted according to the manufacturer's instructions (QIAamp DNA Stool Mini Kit, Qiagen, Valencia, CA). The concentration and purity of the metagenomic DNA were determined using a spectrophotometer (NanoDrop 2000, Thermo Fisher Scientific, Waltham, MA), and the quality of metagenomics DNA was confirmed by agarose gel $(0.8 \%)$ electrophoresis. The resulting DNA was diluted to $1 \mathrm{ng} / \mu \mathrm{L}$ as the template for PCR.

\section{High-Throughput Sequencing of the V3 and V4 Regions of the 16S rRNA gene}

The V3 and V4 regions of $16 \mathrm{~S}$ rRNA genes were amplified using the universal forward primer $338 \mathrm{~F}$ (5'-ACTCCTACGGGAGGCAGCA-3') and the reverse primer 806R (5'-GGACTACHVGGGTWTCTAAT-3') with a set of 6 -nucleotide barcodes (Table 1). Performance of the PCR was described in our previous research (Zhang et al., 2017). Amplified PCR products were detected by electrophoresis in $2 \%$ agarose gels. The target gel was extracted using the QIAquick Gel Extraction Kit according to the kit protocol (Qiagen). TruSeq DNA PCR-Free Sample Preparation Kit (Illumina Inc., San Diego, CA) was used to construct the DNA library. After quantification of the library with a Qubit 2.0 Fluorometer (Thermo Fisher Scientific Inc.) and quantitative PCR, sequencing was conducted using an Illumina HiSeq2500 PE250 platform (Illumina Inc.). The high-throughput sequencing data for this research were deposited in the NCBI database under accession number SRR5684456.

\section{Bioinformatics Analysis of the Sequence Data}

The bioinformatics analysis protocols were described in our previous research (Zhang et al., 2016). Briefly, the sequence data from different samples were separated based on the barcode sequences. FLASH software (V1.2.7, http://ccb.jhu.edu/software/FLASH/ ) was used to assemble reads that were barcode- and primer-free to obtain the raw tags. Qiime pipeline (V1.7.0, http://qiime.org/scripts/split_libraries_fastq .html) contains a suite of software tools that were used to filter out the low-quality tags and retain effective tags for further analysis. Uparse software (Uparse v7.0.1001, http://drive5.com/uparse/) was applied to cluster all effective tags to the operational taxonomic units (OTU) based on 97\% identity of the sequences. Representative OTU with high frequency of occurrence were selected and annotated for taxonomic information using the Mothur method (Wang et al., 2007) and SSUrRNA database in SILVA (http://www.arb-silva .de/) with a threshold of 0.8 to 1. PyNAST software (version 1.2) and the Core Set database in Greengenes (http://greengenes.lbl.gov/) was used to align multiple sequences and analyze phylogenetic relationships among all representative sequences of OTU and determine alpha and beta diversity. Indices of Chao1, Simpson, Shannon, abundance-based coverage estimator, and observed species were calculated by Qiime to study alpha 
Table 1. Sample sequencing information and alpha diversity of bacteria in milk (3 samples each) from Saanen (S) and Guanzhong $(G)$ goats ${ }^{1}$

\begin{tabular}{|c|c|c|c|c|c|c|}
\hline Group & Sample & Goat milk & Raw reads & Qualified & Barcode & Q20 \\
\hline \multirow{2}{*}{ S } & $\mathrm{S} 2$ & \multirow{2}{*}{ Saanen } & 58,766 & 49,737 & TTAGGC & 98.42 \\
\hline & S3 & & 46,682 & 41,017 & TGACCA & 98.58 \\
\hline \multirow{2}{*}{ G } & G2 & \multirow{2}{*}{ Guanzhong } & 31,118 & 26,103 & GCCAAT & 98.58 \\
\hline & G3 & & 45,830 & 40,439 & CGATGT & 98.65 \\
\hline Group G mean & & 682.36 & 4.36 & 0.83 & 548.33 & 680.59 \\
\hline
\end{tabular}

${ }^{1} \mathrm{Q} 20=$ the bases with minimum base call accuracy of $99 \%$ in effective tags. ACE = abundance-based coverage estimator.

diversity (De Nardi et al., 2016; Ji et al., 2017). The taxonomic and phylogenetic tree of bacteria in milk from Saanen goats and Guanzhong goats was presented using the GraPhlAn software (https://bitbucket.org/ nsegata/graphlan/wiki/Home). Phylogenetic Investigation of Communities by Reconstruction of Unobserved States software (PICRUSt, v1.0, http://picrust.github .io/picrust/) was used to predict metagenome functional features from 16S rRNA genes (Langille et al., 2013). Amino acid sequences were aligned and translated from the gene catalog against the proteins (or domains) in the KEGG databases (http://www.genome.jp/kegg/). Relationships between genera, metabolic pathways, and goat milk components were calculated using the Spearman rank correlation coefficient and visualized as a network in Cytoscape software (v 3.2.1; http://www .cytoscape.org/). The R program (v 2.15.3; https:// www.r-project.org/) was used for plotting data and for statistical analysis.

\section{Quantification of the Main Components in Goat Milk}

The quantities of protein, fat, and lactose of goat milk were detected according to the standards (ISO, 2014; AOAC International, 2000; ISO, 2007, respectively). Amino acids were evaluated using the method described by Marino et al. (2010).

\section{RESULTS}

\section{High-Throughput Sequencing of Amplicons}

Using high-throughput sequencing, 265,650 sequences were obtained. The bases with minimum base call accuracy of $99 \%$ in effective tags (Q20) for each sequence amplicon were all greater than $98 \%$, indicating high levels of accuracy in the sequencing data and indicating that they were reliable for further study. The specific sequence information of each sample is shown in Table 1.

\section{OTU Clustering and Taxonomic Annotation of the Goat Milk Microbiome}

To better understand the OTU information and their taxonomic annotation, tags and OTU were calculated and summarized. Using a combination of GraPhlAn (Asnicar et al., 2015) and OTU Table (http://qiime .org/scripts/make_otu_table.html), the taxonomic annotation for the OTU from samples of milk from different breeds of dairy goat was defined and the predominant phyla and genera identified (Table 2; Figures 1 and 2). Using the results of the OTU, a Venn diagram (Figure 3) was created to show the number of OTU of microbes that were shared in the 2 milk samples and the variance between them. The number of mutual OTU in milk samples from the 2 breeds of goat was 802 , representing $44.9 \%$ of all OTU. Of all bacteria,

Table 2. Relative abundance of the main bacterial phylum and genus in milk from Saanen goats (S) and Guanzhong goats $(\mathrm{G})$

\begin{tabular}{lrr}
\hline Taxon & S Mean & G Mean \\
\hline Phylum & & \\
Proteobacteria & 59.37 & 83.24 \\
Firmicutes & 19.06 & 9.51 \\
Bacteroidetes & 3.63 & 1.36 \\
Actinobacteria & 1.03 & 0.25 \\
Genus & & \\
Enterobacter & 33.51 & 15.88 \\
Acinetobacter & 3.17 & 23.33 \\
Pseudomonas & 6.20 & 20.05 \\
Staphylococcus & 5.67 & 2.72 \\
Stenotrophomonas & 0.53 & 5.89 \\
Massilia & 3.60 & 1.28 \\
Bacillus & 0.78 & 2.28 \\
Streptococcus & 0.97 & 0.66 \\
Bacteroides & 1.44 & 0.19 \\
Delftia & 0.05 & 1.54 \\
Lachnoclostridium & 1.46 & 0.09 \\
Escherichia-Shigella & 1.07 & 0.37 \\
Lactococcus & 0.81 & 0.54 \\
Janthinobacterium & 0.89 & 0.45 \\
Sphingomonas & 0.30 & 0.81 \\
Lactobacillus & 0.87 & 0.14 \\
\hline & &
\end{tabular}


53.5 and $73.4 \%$ were specific to milk from Saanen and Guanzhong goats, respectively.

The results showed that the phyla Proteobacteria, Firmicutes, Bacteroidetes, and Actinobacteria were all present at levels of more than $0.5 \%$ of all bacteria; Proteobacteria was the predominant phylum, representing more than $71 \%$ of all bacteria present. We detected a significantly greater abundance of members of the phylum Actinobacteria in milk from Saanen goats compared with milk from Guanzhong goats $(P<0.05$; Figure 4$)$.

At the genus level, Enterobacter, Acinetobacter, Pseudomonas, Staphylococcus, Stenotrophomonas, Massilia, Bacillus, Streptococcus, and Bacteroides (in order from high to low abundance) were present at levels of more than $0.5 \%$ of all bacteria; Enterobacter was the predominant genus, representing more than $20 \%$ of all bacteria present. The genera Blautia, Escherichia-Shigella, Parabacteroides, Bacteroides, Alistipes, Shewanella, Bradyrhizobium, Methylobacterium, Halomonas, Bifidobacterium, Lachnoclostridium, Fecalibacterium, Massilia, Ruminiclostridium, and Chryseobacterium were more abundant in milk from Saanen goats than in milk from Guanzhong goats. In contrast, Pseudomonas, Bacillus, Acinetobacter, Stenotrophomonas, Rhizobium, and Delftia were more abundant in milk from Guanzhong goats than in milk from Saanen goats. The significantly different genera were Ruminococcaceae, Panacagrimonas, Facklamia, Nitrosomonas, and Weissella $(P<0.05)$.

Both probiotics and pathogens were identified in the raw milk. The probiotics included the genera Lactococcus $(0.67 \%)$, Lactobacillus $(0.45 \%)$, Bifidobacterium (0.24\%), Enterococcus (0.18\%), Weissella (0.0076\%), and Pediococcus $(0.0028 \%)$. Some of the lactic acid bacteria present could be identified to the species level: Lactococcus lactis (0.67\%), Streptococcus thermophilus (0.58\%), Lactobacillus delbrueckii (0.21\%), Lactobacillus casei (0.10\%), Lactobacillus intestinalis (0.07\%), Lactobacillus reuteri (0.01\%), Lactobacillus ruminis (0.0083\%), Lactobacillus curvatus $(0.0007 \%)$, Lactobacillus iners (0.003\%), Lactobacillus animalis (0.019\%), Lactobacillus mucosae (0.002\%), Bifidobacterium longum (0.12\%), Bifidobacterium animalis (0.08\%), Bifidobacterium kashiwanohense (0.04\%), Bifidobacterium dentium (0.01\%), Weissella cibaria $(0.008 \%)$, and Pediococcus pentosaceus (0.0028\%). Pathogens were identified as well, including Staphylococcus (4.19\%), Bacillus anthraci (1.52\%), Escherichia-Shigella (0.71\%), Arcobacter cryaerophilus (0.0062\%), Myroides odoratus $(0.0062 \%)$, and Aerococcus viridians $(0.02 \%)$.

Some bacteria are highly resistance to heat, resulting in sterilization failures during pasteurization. Such bacteria were found in the raw goat milk in this study, including Bacillus (1.53\%) and Corynebacterium $(1.00 \%)$. The existence of these bacteria in milk prod- ucts would cause the product to fail quality control. Other bacteria, such as psychrotrophic microorganisms, are heat sensitive but can produce heat-resistant lipase and protease under certain conditions, which can lead to the deterioration of milk products (Liang et al., 2017). These heat-resistant enzymes could remain active following sterilization and even UHT processing. Subsequent catalytic decomposition of milk protein and lipid could lead to flavor variation and loss of nutrients. Psychrotrophic bacteria were found in the current study, including Pseudomonas (13.12\%), Bacillus (1.53\%), Serratia (0.05\%), and Psychrobacter (0.007\%).

\section{Alpha Diversity of the Microbiome in Goat Milk}

Alpha diversity was used as a measure of diversity within the microbial community of each sample (Ai et al., 2017). Five indices were determined (Chao1, Simpson, Shannon, abundance-based coverage estimator, and observed species) to measure the alpha diversity of the microbiome in the sample. The average values of the 5 indices for the microbiome was significantly higher in milk from Saanen goats than in milk from Guanzhong goats $(P<0.05$; Table 1$)$, indicating that the alpha diversity of the microbiome in milk from Saanen goats was higher than that of Guanzhong goats. The rarefaction curve (Figure 5) confirmed the validity of the sequencing data and showed that the abundance of different constituents varied depending on sample.

\section{Beta Diversity of the Microbiome in Goat Milk}

Beta diversity is a comparative analysis of microbial community composition in different samples (Yan et al., 2016). According to the results of taxonomic annotation and abundance information for OTU, the same OTU were classified in the profiling table to calculate the unweighted and weighted Unifrac distances based on the relationship score between OTU. Based on the $t$-test and the Wilcoxon signed-rank test, we found a significant difference in bacterial beta diversity in milk from Saanen goats compared with Guanzhong goats (Figure 6).

Analysis of similarity (ANOSIM) analysis showed that there were greater differences in composition of the microbial community in milk across the 2 breeds than among milk samples from the same breed of goat $(\mathrm{R}=0.2963$, http://cc.oulu.fi/ jarioksa/softhelp/ vegan/html/anosim.html; Figure 7). This confirmed the multiple response permutation procedure (MRPP) analysis, which indicated that differences between groups were higher than differences within group (A $=0.1737 ;$ http://cc.oulu.fi/ jarioksa/softhelp/vegan/ html/mrpp.html). 
Functional Genes of the Bacteria Present and Their Metabolism in Goat Milk

The PICRUSt software was used to predict the functional genes of the bacteria present in goat milk and their metabolic pathways (Table 3). Of the functional genes of microorganisms in goat milk samples, $44.14 \%$ were related to the following metabolic pathways: AA metabolism, carbohydrate metabolism, energy metabolism, nucleotide metabolism, metabolism of cofactors

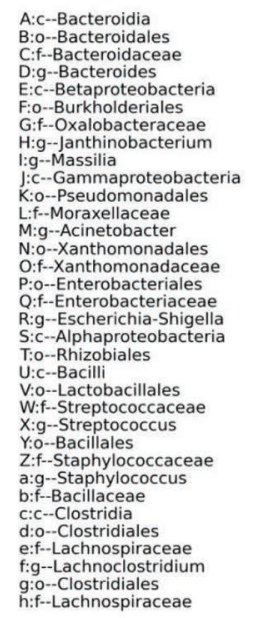

OTU Tree of S by GraPhIAn

P--ACTINOBACTERIA P-BACTEROIDETES P--FIRMICUTES

D: $\mathrm{g}$--Bacteroidacea

(a)

:f-Oxalobacteraceae

C.--Gammaproteobacteri

f--Moraxellaceae

:--Xanthomonadales

:o--Enterobacteriales

R:g--Escherichia-Shigell

:o--Rhizobiales

:0--Lactobacillales

-Streptococcus

g--Staphylococcus

g:o---Clostridiales

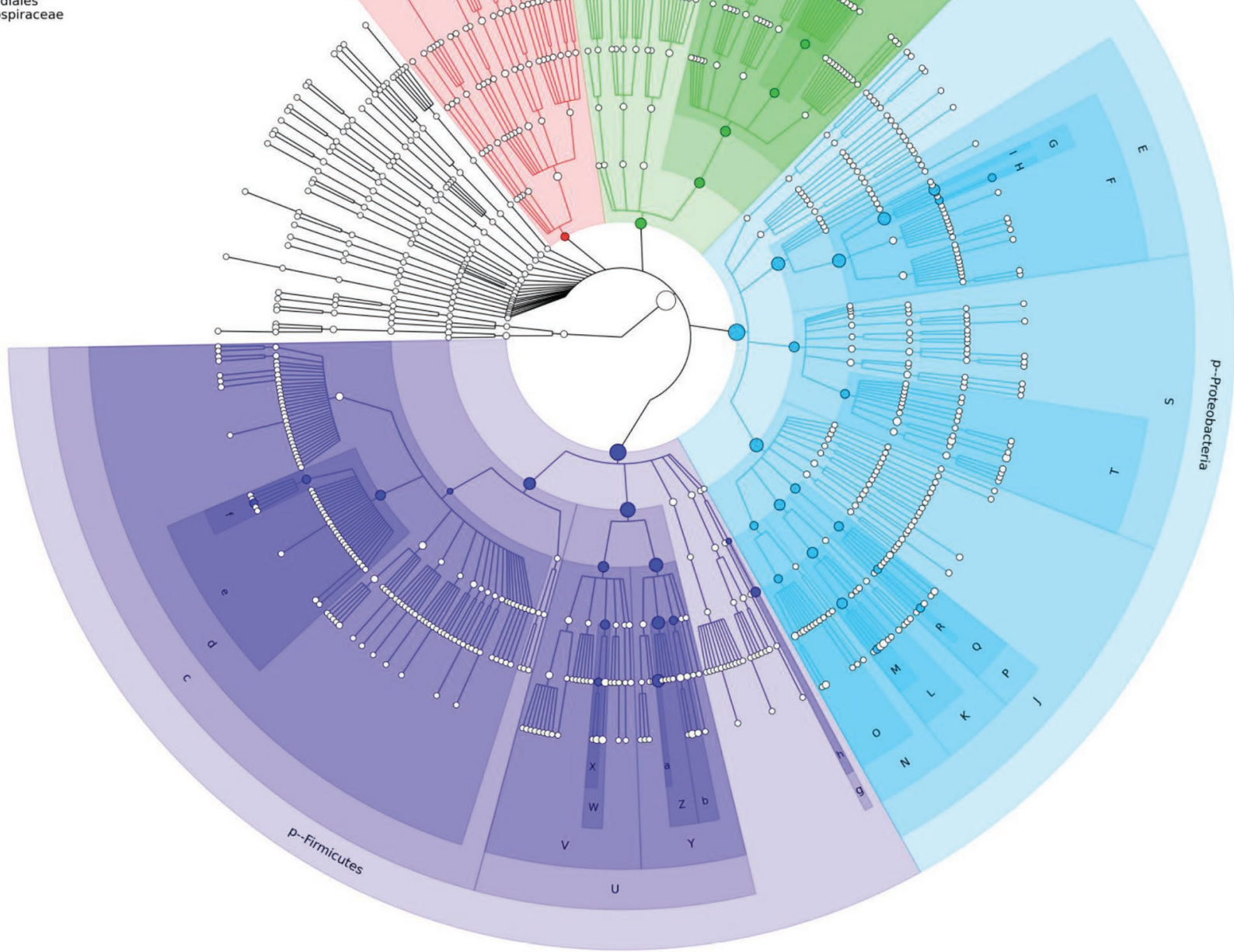

Figure 1. Taxonomic and phylogenetic trees of bacteria in milk from Saanen goats (group S) and Guanzhong goats (group G). OTU = operational taxonomic unit. Color version available online. 
and vitamins, lipid metabolism, xenobiotics biodegradation and metabolism, enzyme families, glycan biosynthesis and metabolism, metabolism of terpenoids and polyketides, metabolism of other AA, and biosynthesis of other secondary metabolites. Genes involved in AA metabolism and carbohydrate metabolism predominated, accounting for 11.93 and $11.23 \%$ of all genes, respectively.

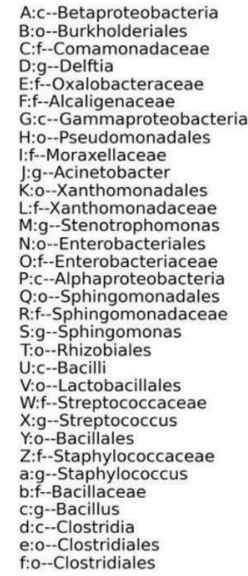

\section{OTU Tree of G by GraPhIAn}

P-BACTEROIDETES P-FIRMICUTES
P--PROTEOBACTERIA

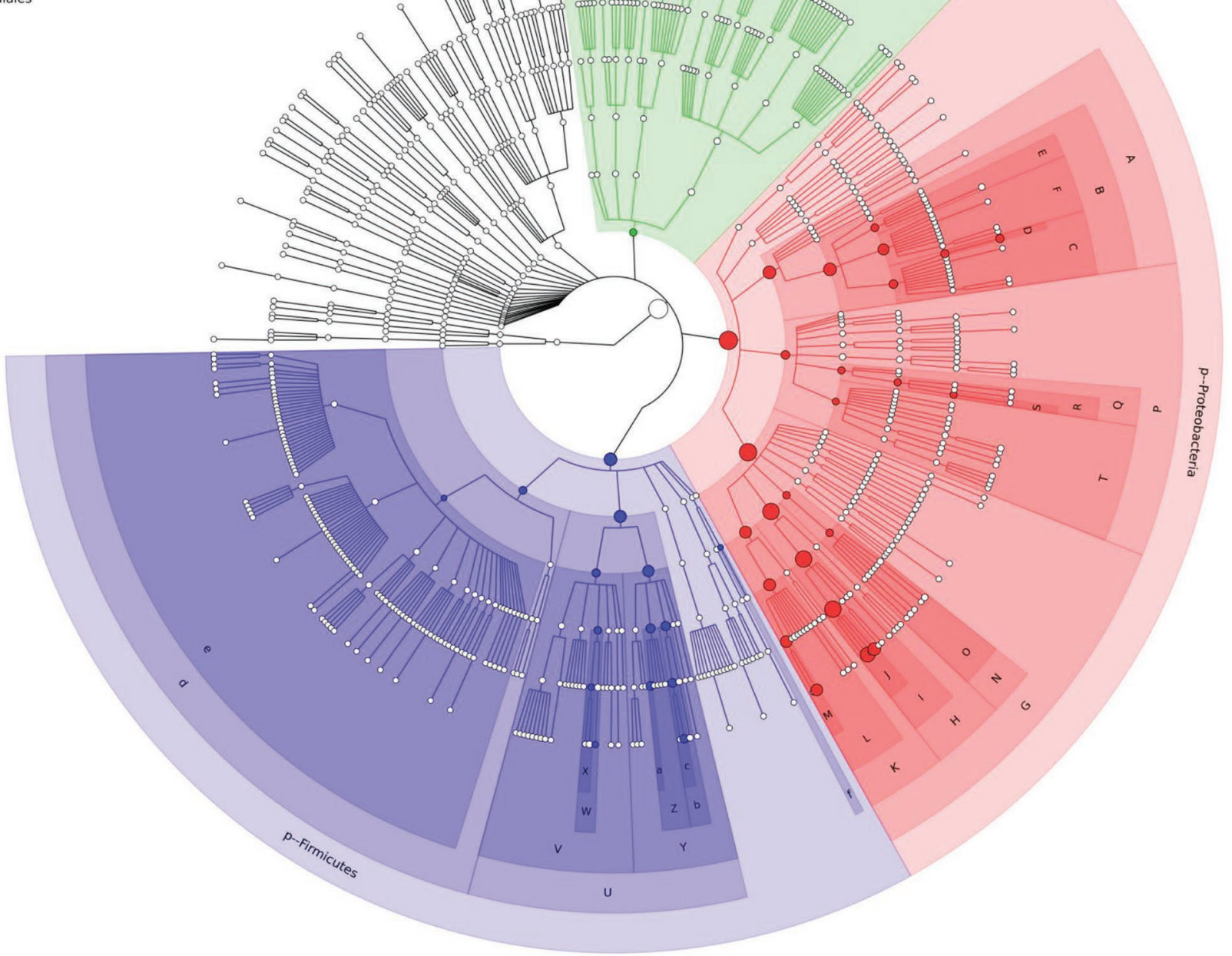

Figure 1 (Continued). Taxonomic and phylogenetic trees of bacteria in milk from Saanen goats (group S) and Guanzhong goats (group $\mathrm{G})$. OTU = operational taxonomic unit. Color version available online. 


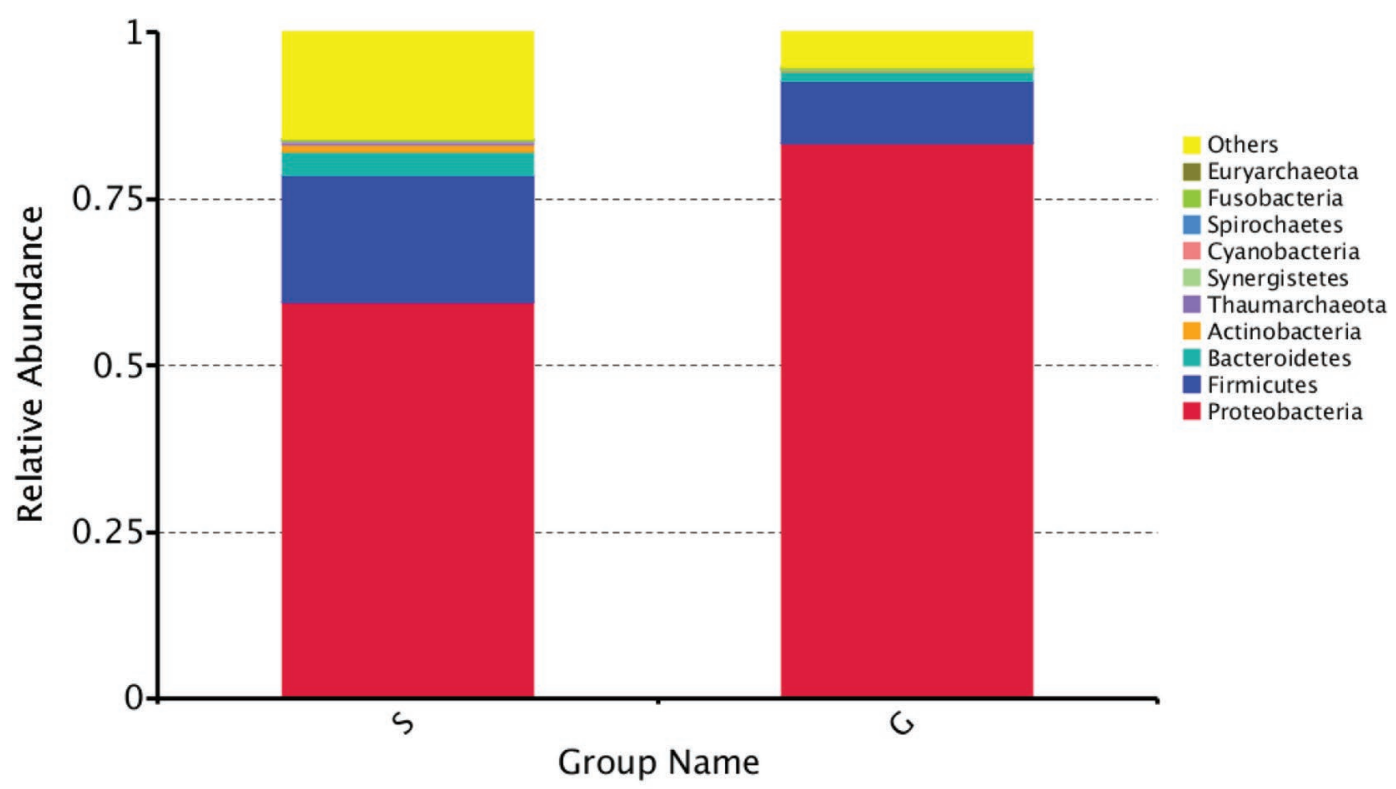

(A)

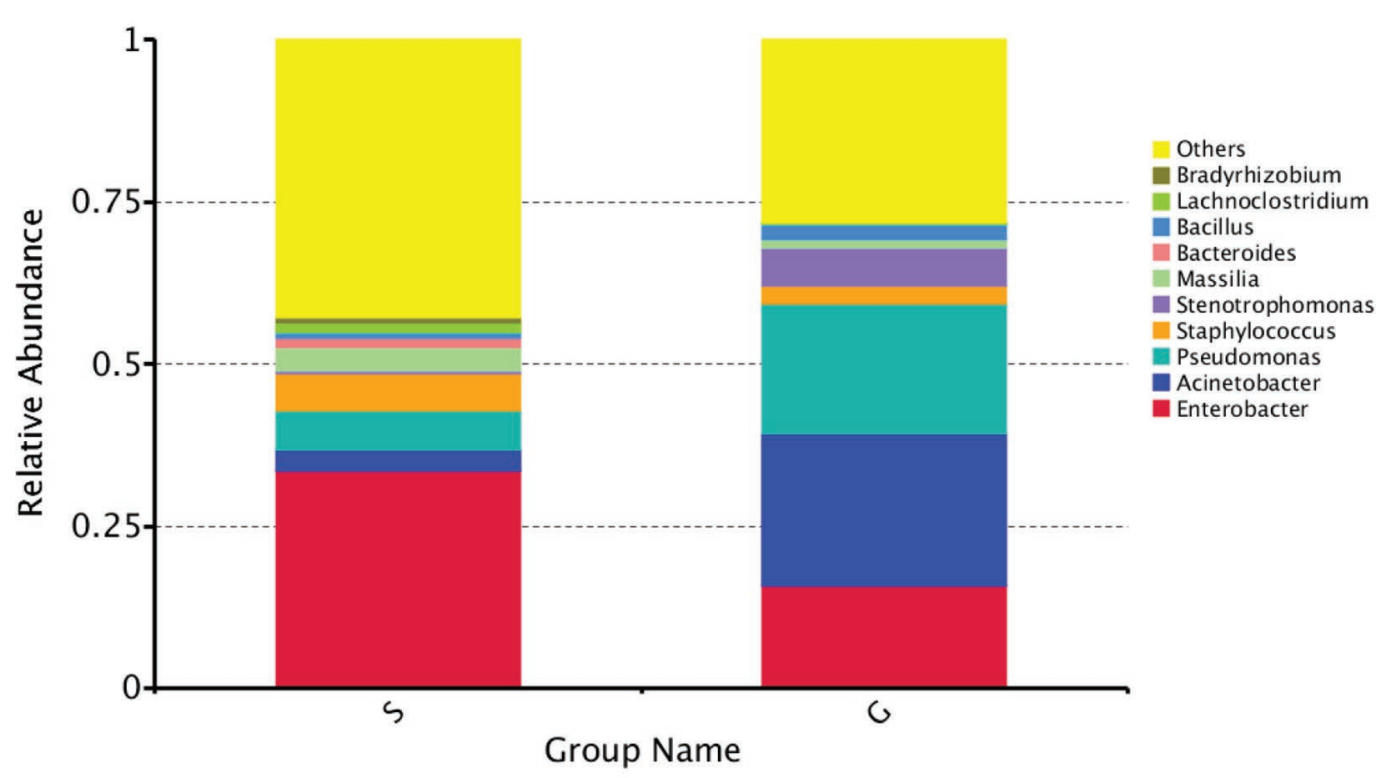

(B)

Figure 2. Relative abundance of bacterial phyla (A) and genera (B) in milk from Saanen goats (group S) and Guanzhong goats (group G). See Supplemental Tables S1 and S2 for plotting Figure 2(A) and Figure 2(B) for further information (https://doi.org/10.3168/jds.2017-13244). Color version available online.

\section{Correlation Between Composition of Goat Milk, Microbial Composition, and Metabolism}

We detected greater quantities of lactose and the AA Asp, Glu, His, Leu, Met, and Thr $(P<0.05)$ in milk from Saanen goats compared with milk from Guanzhong goats (Table 4). Lactococcus, Lactobacillus, Bifidobacterium, Streptococcus, Staphylococcus, Fecalibacterium, Lachnoclostridium, Methylobacterium, and Parabacteroides, which are lactose-utilizing genera, were more abundant in Saanen than in Guanzhong goat milk. Higher levels of lactose in Saanen milk may explain its 


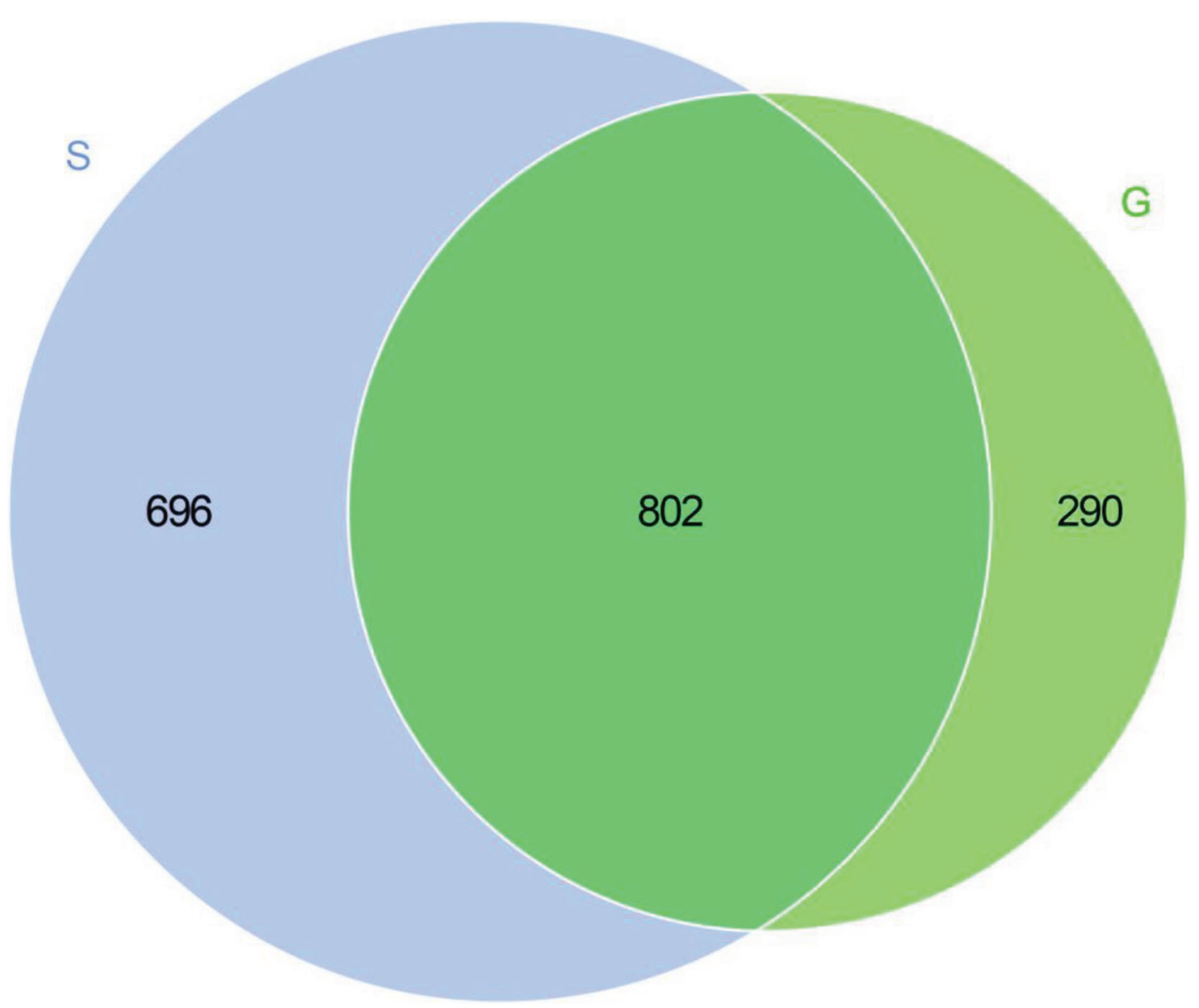

Figure 3. Venn diagram of number of operational taxonomic units of bacteria in milk from Saanen goats (S/blue) and Guanzhong goats (G/green). Color version available online.

greater microbial diversity. The AA Asp, Glu, His, Leu, Met, and Thr were positively correlated with Alistipes, Bacteroides, Bifidobacterium, Blautia, Bradyrhizobium, Brevibacillus, Chryseobacterium, Escherichia-Shigella, Faecalibacterium, Halomonas, Janthinobacterium, Lactobacillus, Lactococcus, Lysobacter, Massilia, Methylobacterium, Parabacteroides, Rhizobium, Shewanella, Staphylococcus, and Streptococcus, which were also enriched in Saanen goat milk (Figure 8).

\section{DISCUSSION}

In this research, microbial diversity in milk from Saanen and Guanzhong goats from the Guanzhong area of China was evaluated using high-throughput sequencing of the V3 and V4 regions of the 16S rRNA gene. The predominant phyla found in raw milk from both breeds were Proteobacteria, Firmicutes, Bacteroidetes, and Actinobacteria, and the predominant genera were
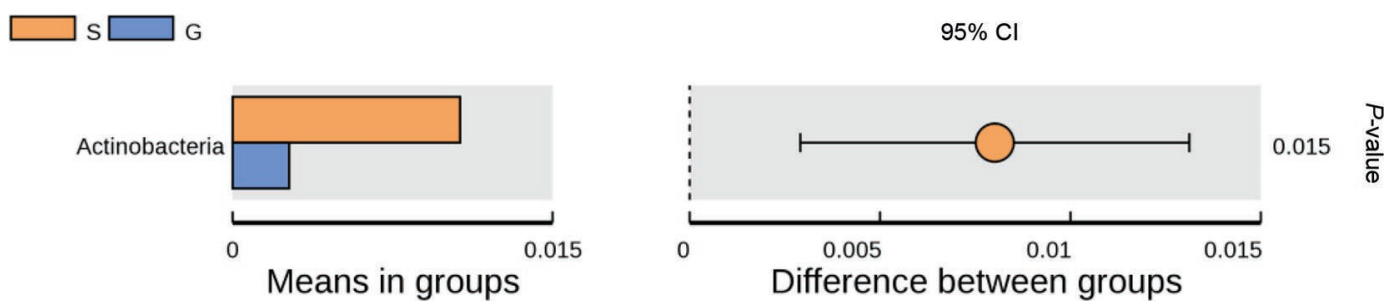

Figure 4. Significant difference in abundance of members of the Actinobacteria phylum present in milk from Saanen goats (S) and Guanzhong goats $(\mathrm{G})$ as determined by $t$-test. Color version available online. 
A

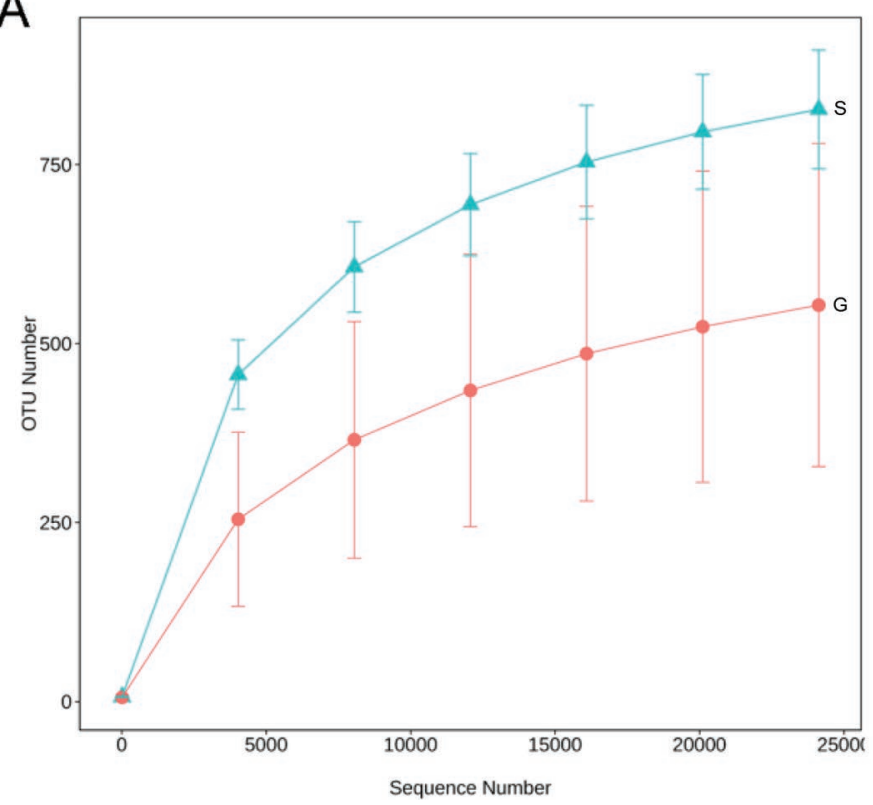

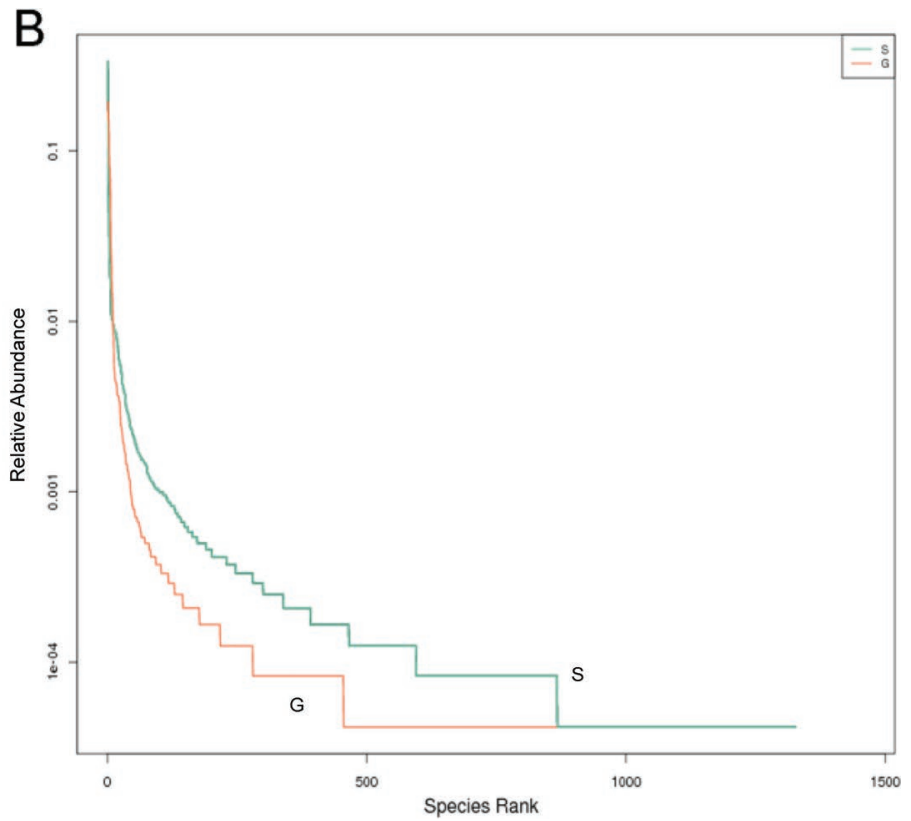

Figure 5. Rarefaction curve (operational taxonomic units, OTU; means $\pm \mathrm{SD} ; \mathrm{A}$ ) and rank abundance curve (B) of bacterial diversity in the Saanen goat milk (S/blue line) and Guanzhong goat milk (G/red line). Color version available online.

Enterobacter, Acinetobacter, Pseudomonas, Staphylococcus, and Stenotrophomonas. Both probiotics and pathogens were identified in the raw milk, which are good resources for isolation of the lactic acid bacteria that could be used in the production of fermented milk products.

Tormo et al. (2011) studied bacterial diversity in milk from goats in the Languedoc Roussillon and Midi-Pyrenees regions of France through PCR-temperature gradient gel electrophoresis (TGGE) and PCR-denaturing gradient gel electrophoresis (DGGE). They found that the genera Staphylococcus, Arthrobacter, and Serratia were the predominant bacteria, and that Lactococcus and Enterococcus were the predominant genera of lactic acid bacteria, findings that are similar to our own research. In another study on goat milk, McInnis et al. (2015) found that the predominant phyla were Proteobacteria, Actinobacteria, Firmicutes, and Bacteroidetes, which is also similar to our results. McInnis et al. (2015) focused on the influence of lactation on microbial diversity and sequenced the $\mathrm{V} 4$ region of the $16 \mathrm{~S}$ rRNA gene, whereas our research considered the potential effect of different components in the goat milk on the microbiota and we analyzed both the V3 and V4 regions of the $16 \mathrm{~S}$ rRNA gene.

Many factors influence bacterial diversity in raw milk, including lactation stage, external environment, region, herd health condition, farm management practices, and differences in the type and components of the milk. For example, Quigley et al. (2013) studied the microbial composition of cow milk and found that Lactococcus, Pseudomonas, Leuconostoc, Prevotella, Fecalibacterium, and Bacteroides were the predominant genera, which

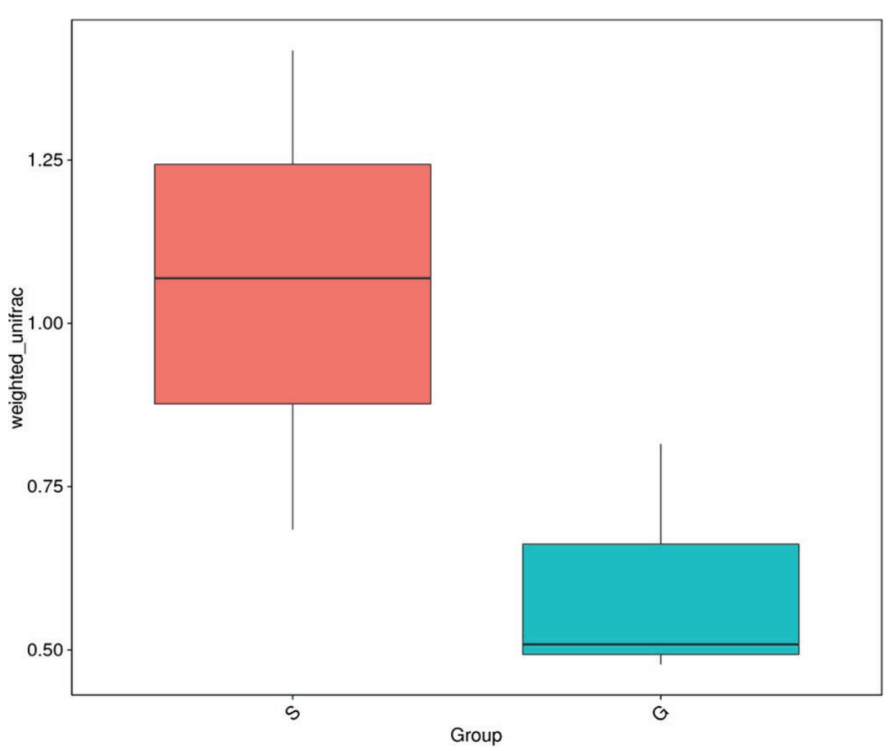

Figure 6. Beta diversity of bacteria in milk from Saanen goats (S) and Guanzhong goats $(\mathrm{G})$ based on Weighted Unifrac distance. The limits of the box (the bottom and top of the box) are the first and third quartiles of the weighted Unifrac distance, and the line (band inside the box) is the second quartile of the weighted Unifrac distance (the median). The ends of the whiskers represent the minimum and maximum of the weighted Unifrac distance. Color version available online. 


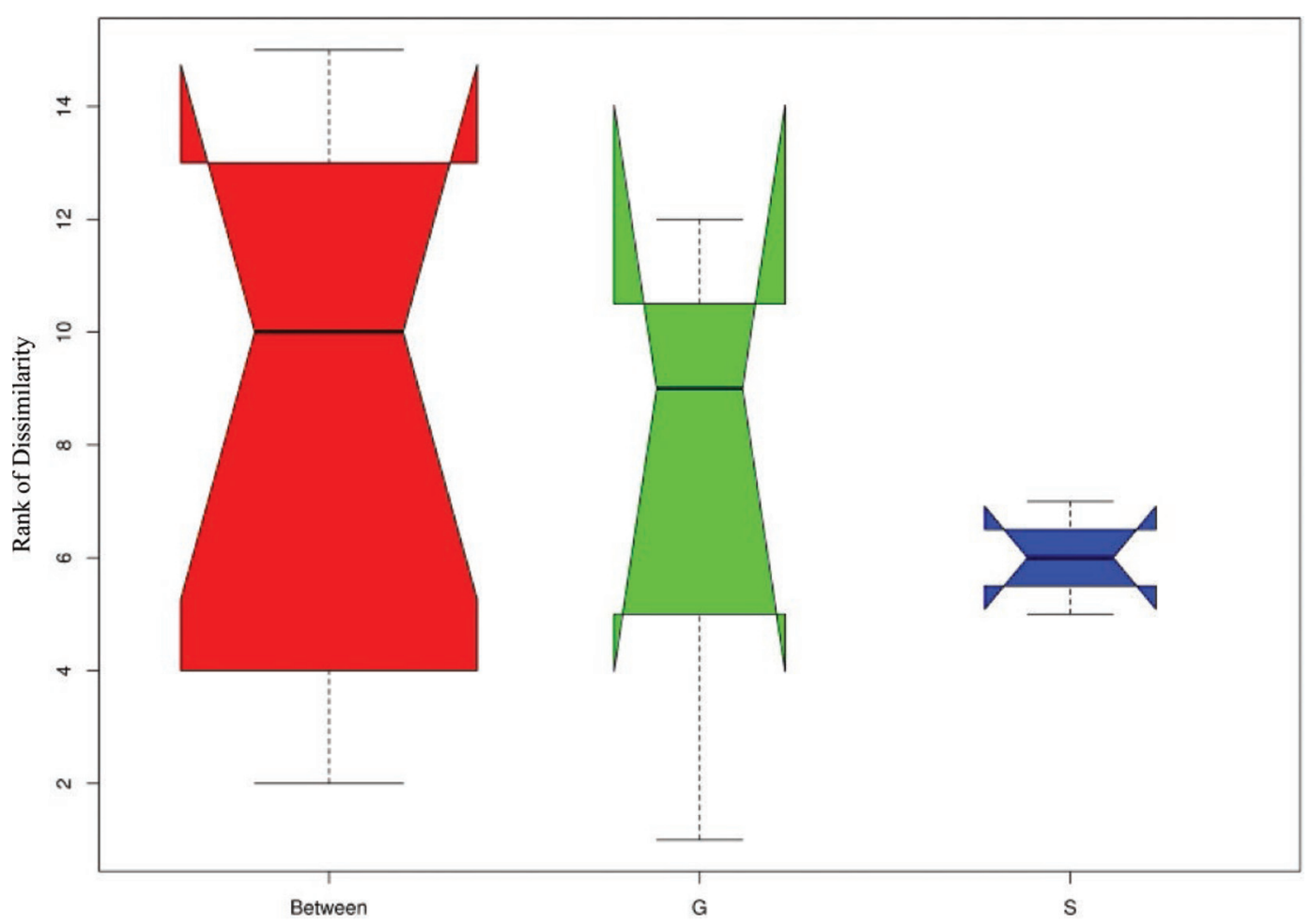

Figure 7. Analysis of similarities (ANOSIM) analysis of the difference in structure of bacterial community in milk from Saanen goats (S) and Guanzhong goats $(\mathrm{G})$. The Y-axis shows the rank of dissimilarity. The ends of the whiskers represent the minimum and maximum of all the data within the group. Color version available online.

was not the same as our study with goat milk. However, among lactic acid bacteria, the predominant genus in all these studies was Lactococcus, which was also the case for studies on camel milk (Ismaili et al., 2016).
Goats and cows vary considerably in their lactation organs, which leads to differences in the composition of their milk. Furthermore, there are more than 50 breeds of dairy goat in the world and components of milk vary

Table 3. The functional features relating to metabolism of genes from bacteria in milk from Saanen goats (S) and Guanzhong goats $(\mathrm{G})$





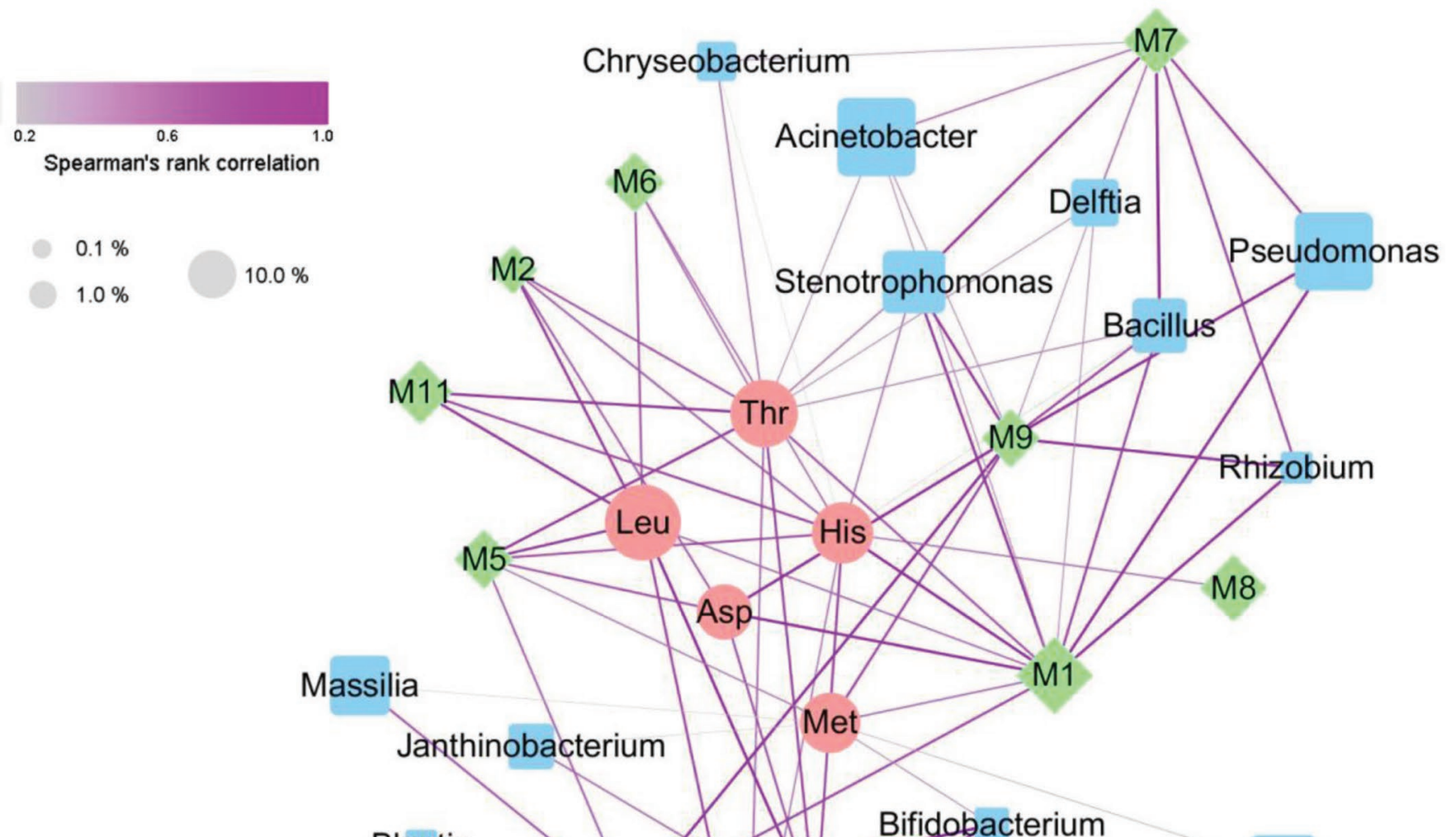

$0.1 \%$

$10.0 \%$

$1.0 \%$

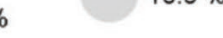

M1 Amino Acid Metabolism

M2 Biosynthesis of Other Secondary Metabolites

M3 Carbohydrate Metabolism

M4 Energy Metabolism

M5 Enzyme Families

M6 Glycan Biosynthesis and Metabolism

M7 Lipid Metabolism

Brevibacillus

Blautia

Bifidobacterium

Escherichia.Shigella

M8 Metabolism of Cofactors and Vitamins

M9 Metabolism of Other Amino Acids

M11 Nucleotide Metabolism

Figure 8. Correlations among predominant bacterial genera, lactose, amino acid preference, and microbial metabolic pathways (M1 to M11) The blue, green, red, and yellow points represent bacteria genera, metabolic pathways, amino acids, and lactose, respectively. Color version available online. 
Table 4. Quantity of moisture $(\%)$, lactose $(\mathrm{g} / 100 \mathrm{~mL})$, fat $(\mathrm{g} / 100$ $\mathrm{mL})$, protein $(\mathrm{g} / 100 \mathrm{~mL})$, and different $\mathrm{AA}(\mathrm{mg} / 100 \mathrm{~mL})$ in milk from Saanen $(\mathrm{S})$ and Guanzhong $(\mathrm{G})$ goats

\begin{tabular}{lrrc}
\hline Component & S Mean & G Mean & $P$-value \\
\hline Moisture & 87.67 & 88.07 & 0.083 \\
Lactose & 4.09 & 3.88 & 0.003 \\
Fat & 3.78 & 3.76 & 0.184 \\
Protein & 3.56 & 3.48 & 0.01 \\
Ala & 105.97 & 100.82 & 0.082 \\
Arg & 112.09 & 116.83 & 0.062 \\
Asp & 244.67 & 224.00 & 0.017 \\
Cys & 11.74 & 8.70 & 0.171 \\
Glu & 775.67 & 712.33 & 0.001 \\
Gly & 69.49 & 67.68 & 0.12 \\
His & 94.67 & 85.67 & 0.023 \\
lle & 175.41 & 178.13 & 0.084 \\
Leu & 361.67 & 312.00 & 0.001 \\
Lys & 289.84 & 293.17 & 0.17 \\
Met & 90.00 & 79.67 & 0.03 \\
Phe & 174.31 & 171.28 & 0.068 \\
Pro & 336.32 & 339.01 & 0.693 \\
Ser & 168.34 & 170.70 & 0.297 \\
Thr & 228.00 & 190.67 & 0.002 \\
Tyr & 134.91 & 133.80 & 0.622 \\
Val & 245.52 & 251.68 & 0.07 \\
\hline
\end{tabular}

with breed, which may also lead to microbial diversity in the milk. In our study, the components of milk from Saanen and Guanzhong goats raised on the same grazing land at the same time were different. From the physicochemical data on milk from the 2 breeds, we detected differences in the quantities of lactose and 6 AA. Amino acid metabolism and carbohydrate metabolism were the main metabolic pathways identified for bacteria in milk from Saanen goats, and the higher levels of lactose and AA in milk from Saanen goats may explain the greater microbial diversity in this milk. The abundance of the genera Streptococcus, Lactococcus, Lactobacillus, Bifidobacterium, Staphylococcus, Fecalibacterium, Lachnoclostridium, Methylobacterium, and Parabacteroides were greater in milk from Saanen goats, which might be expected as these genera can all utilize lactose. We found no difference $(P>0.05)$ in the $\mathrm{pH}$ of the raw milk from 2 breeds ( $\mathrm{pH}$ of 7.09 and 7.10 for Saanen and Guanzhong milk), suggesting that fermentation had not started and that the milk was still fresh. Thus, the presence of more lactose-utilizing genera must be due to the higher levels of lactose present in milk from Saanen goats. The 6 AA were also at higher levels in milk from Saanen goats than in milk from Guanzhong goats, which would account for the greater abundance of genera with a capacity for AA metabolism, particularly Massilia, Bacteroides, Lysobacter, Chryseobacterium, Halomonas, Blautia, and Shewanella. The differences in composition of the milk from the 2 goat breeds contributes to the microbial diversity that each supports, and illustrates the po- tential role of milk composition on the abundance of different bacteria within the microbial community. This confirms the potential for a relationship between milk composition and milk microbial diversity.

\section{CONCLUSIONS}

Many VBNC bacteria cannot be isolated and identified by conventional cultivation methods, and metagenomic-based $16 \mathrm{~S}$ rRNA gene sequencing is a good way to identify these species within food samples. This can help determine whether sterilization of foods is necessary to guarantee their safety. Sequencing of the variable regions of the $16 \mathrm{~S}$ rRNA gene is an effective way to identify bacterial contamination; the method is low cost, high speed, and highly accurate. In our research, probiotics and pathogens were both identified using this method, which could be used to guide the isolation of beneficial bacteria and identify whether there is a need to sterilize the raw milk to guarantee its safety.

\section{ACKNOWLEDGMENTS}

This research was supported by the Fundamental Research Funds for the Central Universities (Grant No. GK201603097 and GK201703063), the Major Transformation of Scientific and Technological Achievements Special Program of Shaanxi Province, China (No. 2016KTG01-02), the Science and Technology Research and Development Program of Shaanxi Province, China (No. 2016NY-207), and the Scientific Research Foundation of Hainan University (No. KYQD1548).

\section{REFERENCES}

Ai, D., R. Huang, J. Wen, C. Li, J. Zhu, and L. C. Xia. 2017. Integrated metagenomic data analysis demonstrates that a loss of diversity in oral microbiota is associated with periodontitis. BMC Genomics 18:1041.

Asnicar, F., G. Weingart, T. L. Tickle, C. Huttenhower, and N. Segata. 2015. Compact graphical representation of phylogenetic data and metadata with GraPhlAn. PeerJ 3:e1029.

AOAC International. 2000. Official Method 989.04: Fat in raw milk. 16th ed. AOAC International, Gaithersburg, MD.

Ayrapetyan, M., and J. D. Oliver. 2016. The viable but non-culturable state and its relevance in food safety. Curr. Opin. Food Sci. 8:127-133.

Ayrapetyan, M., T. C. Willianms, and J. D. Oliver. 2015. Bridging the gap between viable but non-culturable and antibiotic persistent bacteria. Trends Microbiol. 23:7-13.

De Filippis, F., E. Parente, and D. Ercolini. 2017. Metagenomics insights into food fermentations. Microb. Biotechnol. 10:91-102.

De Nardi, R., G. Marchesini, S. Li, E. Khafipour, K. J. Plaizier, M. Gianesella, R. Ricci, I. Andrighetto, and S. Segato. 2016. Metagenomic analysis of rumen microbial population in dairy heifers fed a high grain diet supplemented with dicarboxylic acids or polyphenols. BMC Vet. Res. 12:29.

García, V., S. Rovira, K. Boutoial, and M. B. López. 2014. Improvements in goat milk quality: A review. Small Rumin. Res. 121:51-57. 
Gursel, A., A. Gursoy, E. A. K. Anli, S. O. Budak, S. Aydemir, and F. Durlu-Ozkaya. 2016. Role of milk protein-based products in some quality attributes of goat milk yogurt. J. Dairy Sci. 99:2694-2703.

ISO (International Organization for Standardization). 2007. Milk and milk products-Determination of lactose content by high-performance liquid chromatography. ISO 22662:2007. ISO, Geneva, Switzerland.

ISO (International Organization for Standardization). 2014. Milk and milk products-Determination of nitrogen content-Part 1: Kjeldah principle and crude protein calculation. ISO 8968-1:2014. ISO, Geneva, Switzerland.

Ismaili, M. A., B. Saidi, M. Zahar, A. Hamama, and R. Ezzaier. 2016. Composition and microbial quality of raw camel milk produced in Morocco. J. Saudi Soc. Agric. Sci. https://doi.org/10.1016/j.jssas 2016.12.001

Ji, Y., X. Kong, H. Li, Q. Zhu, Q. Guo, and Y. Yin. 2017. Effects of dietary nutrient levels on microbial community composition and diversity in the ileal contents of pregnant Huanjiang mini-pigs. PLoS One 12:e0172086.

Jia, C. and Z. Wei. 2016. The complete mitochondrial genome of Xinong Saanen dairy goat (Capra hircus). Mitochondrial DNA $27: 3139-3140$

Kibbee, R. J., and B. Örmeci. 2017. Development of a sensitive and false-positive free PMA-qPCR viability assay to quantify VBNC Escherichia coli and evaluate disinfection performance in wastewater effluent. J. Microbiol. Methods 132:139-147.

Langille, M. G. I., J. Zaneveld, J. G. Caporaso, D. McDonald, D. Knights, J. Reyes, J. C. Clemente, D. E. Burkepile, R. L. Vega Thurber, R. Knight, R. G. Beiko, and C. Huttenhower. 2013. Predictive functional profiling of microbial communities using 16S rRNA marker gene sequences. Nat. Biotechnol. 31:814-821.

Liang, X., Z. Meng, L. Zhang, Y. Cui, X. Han, and H. Yi. 2017. The diversity and proteolytic properties of psychrotrophic bacteria in raw cows' milk from North China. Int. Dairy J. 66:23-41.

Marino, R., M. Iammarino, A. Santillo, M. Muscarella, M. Caroprese, and M. Albenzio. 2010. Technical note: Rapid method for determination of amino acids in milk. J. Dairy Sci. 93:2367-2370.
McInnis, E. A., K. M. Kalanetra, D. A. Mills, and E. A. Maga. 2015 Analysis of raw goat milk microbiota: Impact of stage of lactation and lysozyme on microbial diversity. Food Microbiol. 46:121-131.

Quigley, L., R. McCarthy, O. O'Sullivan, T. P. Beresford, G. F Fitzgerald, R. P. Ross, C. Stanton, and P. D. Cotter. 2013. The microbial content of raw and pasteurized cow milk as determined by molecular approaches. J. Dairy Sci. 96:4928-4937.

Silva, F. F. P. D., V. Biscola, J. G. LeBlanc, and B. D. G. de Melo Franco. 2016. Effect of indigenous lactic acid bacteria isolated from goat milk and cheeses on folate and riboflavin content of fermented goat milk. Food Sci. Technol. 71:155-161.

Tormo, H., A. Delacroix-Buchet, C. Lopez, D. A. H. Lekhal, and C. Roques. 2011. Farm management practices and diversity of the dominant bacterial species in raw goat's milk. Int. J. Dairy Sci. 6:29-43.

Wang, Q., G. M. Garrity, J. M. Tiedje, and J. R. Cole. 2007. Naive Bayesian classifier for rapid assignment of rRNA sequences into the new bacterial taxonomy. Appl. Environ. Microbiol. 73:5261-5267.

Yan, X., X. Luo, and M. Zhao. 2016. Metagenomic analysis of microbial community in uranium-contaminated soil. Appl. Microbiol. Biotechnol. 100:299-310.

Yang, B., Y. Wang, and P. Qian. 2016. Sensitivity and correlation of hypervariable regions in $16 \mathrm{~S}$ rRNA genes in phylogenetic analysis. BMC Bioinformatics 17:135.

Zhang, J., X. Wang, D. Huo, W. Li, Q. Hu, C. Xu, S. Liu, and C. Li. 2016. Metagenomic approach reveals microbial diversity and predictive microbial metabolic pathways in Yucha, a traditional Li fermented food. Sci. Rep. 6:32524.

Zhang, J., C. Xu, D. Huo, Q. Hu, and Q. Peng. 2017. Comparative study of the gut microbiome potentially related to milk protein in Murrah buffaloes (Bubalus bubalis) and Chinese Holstein cattle. Sci. Rep. 7:42189.

Zhou, F., Q. Yang, C. Lei, H. Chen, and X. Lan. 2016. Relationship between genetic variants of POU1F1, PROP1, IGFBP3 genes and milk performance in Guanzhong dairy goats. Small Rumin. Res. 140:40-45. 